\title{
Entrelacs
}

Cinéma et audiovisuel

$7 \mid 2009$

L'atelier

\section{La querelle des ateliers cinématographiques}

Depuis l'atelier pédagogique de Luc Moullet

\section{Claudine Le Pallec Marand}

\section{OpenEdition}

Journals

Édition électronique

URL : http://journals.openedition.org/entrelacs/181

DOI : 10.4000/entrelacs. 181

ISSN : 2261-5482

Éditeur

Éditions Téraèdre

Édition imprimée

Date de publication : 1 mars 2009

Pagination : 51-69

ISBN : 978-2-912868-69-5

ISSN : 1266-7188

Référence électronique

Claudine Le Pallec Marand, "La querelle des ateliers cinématographiques », Entrelacs [En ligne],

7 | 2009, mis en ligne le 01 août 2012, consulté le 19 avril 2019. URL : http://journals.openedition.org/ entrelacs/181; DOI : 10.4000/entrelacs.181

Ce document a été généré automatiquement le 19 avril 2019.

Tous droits réservés 


\title{
La querelle des ateliers cinématographiques
}

\author{
Depuis l'atelier pédagogique de Luc Moullet
}

Claudine Le Pallec Marand

"Cinéaste ça veut rien dire, c'est unéaste : celui
qui met les choses ensembles "
Le gendarme en chef dans Le Prestige de la mort

1 L'autoportrait du cinéaste en cinéaste, sur le modèle et l'évolution du motif du peintre dans son atelier (peignant son visage puis son geste de peintre), est une forme contemporaine de l'histoire « de l'émergence du « je » au cinéma »" Dans la théorie, cette représentation tend désormais à supplanter les formes qui la précédèrent: le film de fiction de l'acteur cinéaste (Charlie Chaplin, Orson Welles...), l'affirmation du «je » documentaire, et le film à la première personne basé sur un narrateur-personnage largement déployé par le genre -critique- du film noir².

2 《L'autoportrait cinématographique $»^{3}$ possède aux yeux des spectateurs d'aujourd'hui la force de ce qui n'est pas encore devenu un code, une convention. En enrichissant incontestablement les stratégies discursives disponibles pour mettre en scène la subjectivité au cinéma, il prolonge un mouvement de réflexion qui valorise la vision intérieure, la conscience, la person $a^{4}$, qui voudrait défier la constitution collective inhérente à la plupart des films. Dans cette idée, la transposition de la figure de l'atelier au cinéma permet d'isoler des espaces où résonneraient des traces et des instantanés de sources d'inspiration individuelle - le contexte créateur - mais aussi les phases de mise en œuvre technique et artistique des différents films et œuvres dont la réalisation valorise l'intimité créatrice.

3 Depuis Anatomie d'un rapport, l'œuvre du cinéaste français Luc Moullet, qui se débat « corps et films » avec le genre - jamais défini - du cinéma de l'intime, donne l'occasion, plutôt que d'élire un atelier, de confronter plusieurs possibles de l'atelier cinématographique. Fidèle de «l'intime production» (cinéma à bon marché, en partie auto financé, toujours économiquement contrôlé), tout se passe comme si Luc Moullet, sorte d'adepte de la méthode de Saint Thomas, ne voulait partager et transmettre que ce 
qu'il peut faire voir et entendre: l'ostentation de décors vides, la parole comme accessoire de montage, le gros plan et le regard caméra. Son évangile ${ }^{5}$ ne fait état ni de la mort ni de la résurrection du cinéma selon un atelier qui vaut moins comme une thématique ou une parabole individuelle que comme une école artistique au passé burlesque et à l'avenir libre des bobines ${ }^{6}$.

Le plus souvent, Luc Moullet est évoqué sous les traits d'un géographe et d'un sportif (alpiniste, cycliste, cinéaste des hauteurs...), ou bien d'un économe, au risque que l'on dût déduire de l'homme son atelier plutôt que de (re)construire l'atelier de ses films. A partir de Luc Moullet, on a pu parler d'atelier imaginaire des montagnes, isolant la figure du milieu de l'environnement autobiographique, et d'atelier économique, basé sur "l'économie de moyen " comme le tout de son savoir-faire. Dès lors, comment redéfinir l'atelier Luc Moullet en dehors de ses codes opératoires? En renversant le dernier principe de l'atelier économique, de l'économie vers le savoir-faire, on retrouve le sens pédagogique du terme d'atelier (le sens le plus récent selon le dictionnaire historique de la langue française). Le rôle de la matrice éducative dans la mise en scène suggère une attention particulière au geste cinématographique ${ }^{7}$ en accord avec la tradition humaniste de la Renaissance qui défend la volonté et la capacité de l'artiste contre la toute-puissance de l'outil. En termes d'option cinématographique, Luc Moullet accorde davantage d'attention à l'écriture, au jeu et au montage qu'aux mouvements des images. Il privilégie visiblement (mais non tautologiquement) les changements à vue (changements d'échelle de plans, logique de la parole comme support dominant du montage) dans un dispositif ${ }^{8}$ frontal hérité du burlesque.

5 Le cinéaste fait comme s'il n'y avait rien que le spectateur ne puisse pas voir : les outils (caméra et pellicules dans le champ) et l'équipe technique (interventions sonores ou visuelles) sont intégrés à une œuvre où il est incertain de différencier ce qui procède du documentaire ou de la fiction. Au risque de subir les foudres (ou le sourire) du cinéaste lui-même, que l'on peut aller jusqu'à soupçonner de tentation pataphysique ${ }^{9}$, nous voulons dépasser une dialectique de l'atelier (thèse d'une économie d'échelle artisanale/ antithèse de l'autoportrait du cinéaste au travail) pour dévoiler la bataille artistique et critique de la figure de l'atelier cinématographique comme une lutte confrontant la subjectivité et le savoir-faire transmissible. La figure de l'atelier nous rappelle ainsi qu'une esthétique particulière (pas nécessairement nouvelle) ne s'explique exclusivement ni par des raisons techniques ni par des raisons économiques. Ces raisons sont autant d'entrées dans la construction des films ${ }^{10}$, sans jamais devoir abandonner les pistes de réflexions qui font de l'économie une esthétique et de l'esthétique une affaire de pédagogie ${ }^{11}$.

6 Luc Moullet cinéaste d'un lieu à ciel ouvert, la montagne, y compris les roubines ${ }^{12}$, est une image difficilement réductible à la figure d'un atelier. La montagne est plutôt de l'ordre de la matrice, c'est un réservoir d'inspiration continue, d'images et de paroles: décors, objet-sujet de panoramiques, métaphore, anecdote... « quand je fais autre chose que mes films, je travaille facile ; c'est comme monter un sommet à pied après un col en vélo (...) je sens un chemin en corniche sur le vide, le dialogue entre Rachel qui marche vite et son amie rencontrée là, en tenue de ville ». (Les minutes d'un faiseur de film). Pourtant, Luc Moullet est loin de ne filmer que la montagne. A une critique de commande sur les paysages, il répond par le «tout solaire» du tournage dans ses appartements ${ }^{13}$ et en profite pour proposer un abcédé $e^{14}$ sur le tournage en montagne : les points cardinaux pour la lumière, le repérage dans un site difficile d'accès, la lecture des cartes IGN, les acteurs 
dans le paysage de montagne, l'originalité du lieu, l'écho et la voix off... L'atelier Luc Moullet est donc un univers nomade, c'est-à-dire moins un lieu défini qu'un mouvement entre la ville et la montagne. En ville, on peut plus facilement poser devant l'objectif une caméra et une bande de défilement de la pellicule imprimée (Anatomie d'un rapport) pour montrer cet ancien moyen de révision et de visionnement des pellicules.

«Francis est un géographe méticuleux et un grand connaisseur du cinéma, mais il le traite d'une certaine façon. Il fait des images sans recherche. Il a opté pour une sorte de "lumpen-cinema ». Il a très vite compris que le cinéma avait brûlé l'essentiel de ses cartouches. Le sublime de Murnau, la perfection de Chaplin, la mécanique de Lang appartiennent au passé, ne serait-ce que pour des raisons économiques. Inutile de faire semblant. Avouons nos limites et sachons en rire. ${ }^{15}$

7 Luc Moullet cinéaste $\operatorname{artisan}^{16}$ : cette idée semble être un acquis du paysage cinématographique, voire un lieu commun pour quiconque s'intéresse à ce cinéaste atypique toujours en activité. Du fait de son premier compte rendu dans les Cahiers jaunes en 1956 et de sa première réalisation, le court métrage intitulé Un steak trop cuit, produit dès 1960, selon la loi du genre de la Nouvelle Vague par Georges de Beauregard, Luc Moullet possède un statut particulier. Plus tardif et moins prolixe en longs métrages que les célèbres cinéastes du mouvement, réalisateur de nombreux formats courts, Luc Moullet était devenu de facto un auteur dont chaque film demeure un prototype économique, un exercice d'atelier de production.

8 Trois raisons principales nourrissent la définition de son œuvre par l'analogie artisanale. Ses propres propos et ses activités corporatives (il a participé à la création de la SRF en 1968 après les Etats Généraux du cinéma) le désignent comme un artisan, "un contrebandier de la pellicule ». Secundo, la première rétrospective française de ses films a consacré le principe à travers une déclaration dont il est créditée : "Je suis burlesque à force d'être démuni $»^{17}$. Enfin, la reconnaissance et l'ancrage de l'économie du cinéma dans les études cinématographiques universitaires d'aujourd'hui permettent de réévaluer l'orientation de ses critiques les plus hétérodoxes comme son article daté de septembre 1959, Le Martyr de San Sebastian ${ }^{18}$.

9 En effet, Luc Moullet analyse le festival éponyme en se distinguant d'une critique de la programmation reléguée en seconde partie. Prenant ses distances avec le genre défini à cette occasion comme celui des « comptes rendus de Festival », l'article est introduit par une note liminaire qui justifie la publication de «ce texte qui semble n'avoir avec la critique cinématographique qu'un lointain rapport». Le cinéaste inaugure dans cet article une sorte de structuralisme de la critique de la critique en s'interrogeant sur le genre de la « critique de festivals ». Il en dévoile les attendus implicites superflus, à savoir les notes géographiques (la ville d'accueil), les jugements sur l'organisation et, en dernier ressort, la valeur du festival. Plus surprenant encore, il expose la place économique de la manifestation à l'échelle de la ville, du pays, et enfin de la presse dans l'économie française. Cette échappée culmine dans l'anecdote du confrère marxiste des Lettres françaises qui profite des conditions royales offertes. Le confrère en question devient ainsi un spécimen burlesque d'exploité-exploiteur de l'industrie cinématographique qui anticipe les exploités-exploiteurs consommateurs et ouvriers des chaînes agroalimentaires de Genèse d'un repas. C'est cet article qui fut salué par Jean Douchet ${ }^{19}$ en 2006, à l'occasion de la sortie de la première édition de films du cinéaste en DVD. Le critique nourrit à son tour, et avec talent, la prégnance tenace de la métaphore économique pour cerner l'œuvre de Luc Moullet, sous l'aspect inédit de La jauge. 
10 Au-delà de l'harmonie existant entre son écriture critique et son œuvre de cinéma, Luc Moullet a également évoqué les raisons financières de son engagement dans la production. Il explique qu'il est devenu producteur pour produire son premier long métrage ${ }^{20}$ mais aussi parce que la production de films des autres peut être l'occasion d'un travail rémunéré. Producteur dès 1966, il a financé notamment en 1971 Nathalie Granger de Marguerite Duras.

11 Si la valeur de son attention peu commune à la production est évidente dans la genèse de son cinéma, il n'est pourtant pas inintéressant de s'arrêter à l'une de ses remarques publiées en $1981^{21}$. Luc Moullet s'exprime avec regret et nostalgie à propos du mythe du réalisateur hollywoodien intégré au système des studios, c'est-à-dire ni auteur du scénario, ni technicien, ni surtout producteur. Il apparaît alors qu' « Hélico et grues $»^{22}$, étrangers à l'économie cinématographique légendaire de Luc Moullet «sont les bienvenus » a priori. Il s'agit alors, en s'écartant de la providentielle construction a posteriori d'une téléologie à partir de la spécificité de ses articles critiques, de contourner l'évidence de la position de réalisateur-producteur de Luc Moullet.

12 En dehors de leur production et de leurs décors épurés, comment aborder les trois longs métrages du cinéaste depuis le tournant documentaire de son œuvre, qui veut désormais qu'il joue le rôle principal? Afin de contribuer à l'étude esthétique des films de Luc Moullet, il ne s'agit pas d'énoncer la figure d'un atelier, la pédagogie, pour en nier d'autres possibles: l'atelier comme lieu de création (nomade et quotidien, l'espace à double usage de l'appartement où vit par ailleurs le cinéaste au moment du tournage de chaque film) et l'atelier comme artisanat, contre le Studio (« l'atelier» du travail collectif et de l'empire industriel Hollywoodien). Il s'agit bien de suggérer que ces dernières figures, déjà repérées pour valoriser l'œuvre, ont masqué une figure plus ample, moins conventionnellement attachée au cinéaste : l'atelier pédagogique.

13 L'atelier pédagogique selon Luc Moullet, permet de dépasser une tonalité, le minimalisme, trop aisément rabattable à une affaire d'économie du cinéma, au risque de marginaliser la transmission, tout en humour, d'un savoir-faire. « Luc Moullet scénariste pense-t-il les scènes avec l'expérience de Luc Moullet producteur? $»^{23}$. Oui, mais encore. C'est aussi rappeler l'idée qui veut que s'intéresser à un auteur ${ }^{24}$, selon la dénomination chère à cet ancien critique régulier des Cahiers, c'est comprendre qu'il engage autant une responsabilité économique qu'une option de cinéma. Les limites de cet atelier pédagogique seront bien évidemment celles de son cinéaste, comme en témoigne les tentatives du personnage de Luc Moullet pour faire retarder l'un de ses tournage sous prétexte d'un vent et d'un soleil hostiles au son et à l'image, alors que l'un de ses précieux techniciens d'extérieurs l'apostrophe à l'image : «c'est pas ton boulot !»: Ma première brasse est le seul film de Luc Moullet où apparaît à l'écran le modeste collectif du tournage.

14 De manière récurrente, le personnage de Luc Moullet, qui n'est plus seul en scène, est constamment isolé dans le plan. Bien qu'il se filme sous les traits d'un messie, habillé de coton blanc, chaussé de sandales, un bâton à la main, menant son équipe sur les chemins escarpés d'un raccourci et lui offrant pour chaque station, souffle et parole sur la genèse de l'histoire du film en train de se faire, son personnage est contrarié, dans sa lâcheté, par l'intransigeance des autres professionnels. Les personnages de son équipe récusent les arguments techniques qu'il met en avant, et prennent en charge scrupuleusement et sadiquement le régime alimentaire nécessaire à son jeu d'acteur. Qui doit jouer sa première brasse, ne peut pas manger ! Le choix du cinéma burlesque de Luc Moullet qui 
consiste à se mettre en scène en victime se traduit sans surprise par l'incarnation humoristique d'oppositions au labeur cinématographique : résistance physique du corps et des éléments, résistance humaine de l'équipe. Chez Luc Moullet, il n'est pas question du savoir-faire d'un atelier professionnel collectif comme Numéro Deux de Jean-Luc Godard a pu inscrire la représentation du travail et de l'usine depuis le documentaire et le cinéma militant jusqu'à une réflexion narrative, personnelle, énonciative, matérielle (cinéma/ vidéo) sur l'usine cinéma.

Incontestablement, Anatomie d'un rapport, le quatrième long métrage de son auteur, infléchit la critique de l'œuvre du cinéaste jusqu'à instaurer rétrospectivement une seconde veine dans son cinéma comique. L'énoncé de ses films est désormais susceptible d'être saturé par les "effets de subjectivité $»^{25}$. Si dès son premier court métrage, le cinéaste fait de brèves apparitions, voire des rôles secondaires, le film coïncide avec un statut différend de l'image de son propre corps, puisque l'auteur réalisateur joue désormais son personnage principal. Pour Genèse d'un repas, il est permis d'hypostasier son travail d'enquête documentaire comme structure de base du film. Dès l'introduction, il incarne le contrechamp systématique du film, relayé ensuite par sa voix off. La coïncidence entre le je devant la caméra (et/ou derrière la bande son) et l'introduction de "l'effet documentaire» dans l'œuvre est d'ailleurs un marqueur historique de la décennie : Numéro Deux (Jean-Luc Godard et Anne-Marie Miéville, France, 1975), News from Home (Chantal Akerman, Belgique, $16 \mathrm{~mm}, 1976)$, et Ce répondeur ne prend plus de message (Alain Cavalier, France, $16 \mathrm{~mm}, 1978)$. Dans les années soixante-dix, l'impact cinématographique de l'intrusion énonciative du cinéaste, chère à la modernité, se couple avec son inscription intime et corporelle.

Dans Anatomie d'un rapport, l'acteur principal - auteur-réalisateur-producteur -, en personnage d'alter égo cinéaste, est en prise avec les conséquences de la co-réalisation d'un film sur l'intimité, avec sa compagne, Antonietta Pizzorno. De manière inédite, comme le laisse suggérer la double fin du film, la co-auteure met en scène, sans que Luc Moullet n'intervienne, les scènes du personnage féminin seul à l'écran, et les scènes collectives entre femmes. C'est ce parti pris inédit, d'une écriture commune des scènes communes, et d'une écriture et d'un tournage à deux têtes selon le sexe des personnages, qui se transforme en leçon de mise en scène associative. Pour Luc Moullet, ce film introduit la question de l'autoportrait au long cours, qui va se développer, dès lors, plusieurs œuvres durant. Luc Moullet se met désormais en scène avec ses travaux (artefacts et autocitations : affiches de films, constitution de press-book, mentions orales des films précédents comme variante importante de l'effet de signature en plus de ses apparitions du cinéaste comme corps, voix et pulsations cardiaques), ses outils, ses attributs (caméra, machine à écrire) et ses familiers: dans cette petite compagnie, on reconnaît les partitions et directions musicales de Patrice Moullet, les interprétations de sa compagne Antonietta Pizzorno, et le retour, d'un film à l'autre, de quelques acteurs, comme Marie Christine Questebert, actrice principale de Billy the Kid et d'Anatomie d'un rapport, interviewée dans Ma première brasse et jouant un petit rôle dans Le Prestige de la mort.

Dans le cadre d'une réflexion sur la subjectivité au cinéma et le portrait visuel du cinéaste, le détour par une attention non plus exclusivement monographique, permet de mesurer l'ampleur de la pratique de Luc Moullet dans la matrice d'un cinéma pédagogique comme outil de réhabilitation de l'instance de l'auteur au cinéma. Le versant technique de référence correspond aux évolutions techniques d'après-guerre, dans le 
sens de l'autonomie et de la légèreté du direct (à ne pas confondre avec l'immédiateté du reportage de télévision), au profit de la « libération » du cinéaste de son équipe, jusqu'à la caméra DV des années 90. A l'étranger, la comparaison avec le cinéaste italien Nanni Moretti ${ }^{26}$ est une note de rigueur. Dans sa précieuse thèse universitaire L'autoportrait cinématographique, Muriel Tinel $^{27}$ isole exclusivement six films contemporains pour développer sa réflexion:

« Partant du rapport du cinéaste à sa technique, on rejoindra la question du travail du support qui est sous-jacente depuis le début du propos et qui est aussi l'hypothèse générale de [Raymond] Bellour dans L'entre-images: c'est de l'interaction des médias que naissent des formes inédites. Deux branches indépendantes d'analyse jailliront de cette même idée en forme d'hypothèse : ce n'est pas seulement l'apparition de l'artiste qui compte mais aussi la nature et le travail de cette image. D'un côté on s'interrogera sur l'expérimentation à l'œuvre dans la rencontre de ces supports (superposition, passage, scintillements, montage, arrêt, répétition, vitesse) et leurs conséquences (intrusion de l'autobiographie, disparition de la figure, question de la modernité, visibilité du matériau). De l'autre, on se demandera si finalement l'invention d'une technique de l'image (un procédé ou un effet) n'est pas le lieu privilégié de l'autoreprésentation : parce qu'il est facile de se prendre pour modèle, que cela permet de préserver l'intimité de l'expérience et de s'approprier le dispositif. $»^{28}$

La pertinence de cette thèse permet nettement de distinguer l'autofiction (mise en scène d'un personnage de cinéaste sous les traits de l'acteur réalisateur ou acteur alter égo), le journal intime (mettre en scène son intimité), l'autobiographie (l'approche rétrospective des autobiographies romancées et des autobiographies revendiquées qui se donnent en tant que genre à l'intérieur du genre documentaire), et enfin l'autoportrait cinématographique (" qu'en est-il de moi maintenant dans le cinéma? »).

Mais cette étude survole à peine Luc Moullet dont elle ne cite que Les minutes d'un faiseur de film en omettant $M a$ première brasse, dont le titre masque partiellement la métadiscursivité cinématographique. Il faut sans doute comprendre cette mise à l'écart par le refus du cinéaste de travailler son support pellicule et/ou vidéo et ses compositions plus attentives au montage qu'à la plastique, choix que l'auteure associe implicitement à des pratiques en quelque sorte "régressives " d'autofiction et de simplicité. Or, tout le cinéma de fiction de Luc Moullet ne parle que de faire des films.

Quand elle cite Les minutes d'un faiseur de film, Muriel Tinel décrit une posture : le cinéaste en robe de chambre devant sa machine à écrire. La théoricienne ne voit que l'intimité d'un cinéaste au travail quand le découpage logique du début du film assène davantage d'information. Le premier mouvement, sur le générique du film, est un zoom avant d'un plan général fixe à un gros plan isolé. Le mouvement se déplace du cinéaste, une lettre à la main (la subjectivité), à l'objet d'une leçon sur le cinéma (la lettre postée par sa main). Cet écart explique l'incongruité de l'introduction d'un tu imaginaire dans le film. C'est sans doute à lui que s'adresse cette affirmation énigmatique au milieu d'autres informations (infarctus d'un père, arrosage des plantes) : "J'ai soixante-deux idées de scène principale, je les classe pour avoir une idée de suite logique ».

Or, tout le reste du film va démontrer une leçon, bien connue du cinéma burlesque, que le règne partial de l'imagination (le cinéaste aimerait noter ses rêves) ne permet pas la suite logique. Ainsi, si le nombre de pages blanches placées devant Luc Moullet dans son film est un principe économique, une surface d'écriture et de longueur a priori du film, ce principe semble assez hypothétique pour délimiter une scène, un bon mot, un cadre humoristique. L'impossibilité de relayer les indications chronologiques (la triple scansion 
des gros plans du réveil matin) à une construction du film par séquence privilégie le principe du montage et des chutes narratives, les décisions qui font se succéder un plan à un autre et une valeur de plan à une autre, sans qu'un rythme ou une clôture narrative chapote le film entier. Le film pense en se modelant sur le discours verbal plutôt qu'à partir de la maîtrise technique. A l'aide de la parole, de la musique diégétique du radio cassette, d'un principe imaginatif (l'intrusion du quotidien dans le fantastique), l'expression «j'ai cette scène dans la peau » précède symptomatiquement un léger regard caméra à peine perceptible. En définitive, tout le film illustre l'imaginaire décousu des « pages par jour à la sortie du lit ».

L'attention affichée portée au dispositif ${ }^{29}$ par Muriel Tinel privilégie la problématique de l'atelier à l'échelle de l'histoire de l'art, avec la peinture et la photographie, et isole des figures de mémoire, individuelle et collective, du cinéma et du cinéaste. Peut-on poser l'hypothèse qu'elle ne mesure pas la volonté didactique et la particularité de l'atelier de l'œuvre du cinéaste Luc Moullet? Rivée au dispositif et à la confrontation des natures d'image (photographie, cinéma et vidéo, selon le sous-titre de l'essai de Raymond Bellour), la valorisation de l'histoire de l'art dans la figure de l'atelier éclipse d'autres options de cinéma, pour ne plus se préoccuper de mise en scène qu'exclusivement en terme de subjectivité (cf. chap. VI), notamment des configurations de l'énonciation, et en terme de rapport entre le sujet autoportraitiste avec le média cinématographique (cf. chap. VII).

Dès lors, il n'est pas étonnant que l'auteure se trompe (au regard de ses propres critères) quand elle décrit Anatomie d'un rapport comme un journal intime où les deux réalisateurs jouent leur propre rôle. Or, le nom de Luc Moullet n'est jamais nommé dans ce film et son actrice principale n'est pas la compagne co-auteure, Antonietta Pizzorno, qui intervient plusieurs fois dans le film. Le thème de la sexualité dans Anatomie d'un rapport n'équivaut pas obligatoirement à la forme du journal intime mais débouche sans équivoque sur une question liminaire de cinéma: comment écrire un film à deux en respectant deux discours?

De même, les effets de subjectivité des deux longs métrages suivants ne masquent pas une réflexion pour penser la pratique du cinéma. Génèse d'un repas, documentaire-enquête à la première personne, évoque les problèmes éthiques de la fabrication du film et la déconstruction des logiques d'informations disponibles. Prestige de la mort, autofiction prononcée, reconstruit le système économique et la place de la télévision dans le cinéma français, et la difficulté de faire des films depuis les repérages, la production jusqu'à la diffusion post mortem. Le film dans le film n'est pas, dans Prestige de la mort, un processus métadiscursif moderne mais le tournage d'une adaptation littéraire chère au cinéaste que la pénurie économique n'empêche pas de donner traces à travers d'amples mouvements de caméra et une photographie d'inspiration romantique ${ }^{30}$, singulière dans toute son œuvre.

Ma première brasse est une réponse tout en humour aux figures de l'autoportrait cinématographique discriminées par la thèse de Muriel Tinel. En fait de mémoire collective et cinéphilique, Luc Moullet se travestit en Christ et il compare son bain au goût pour l'eau de James Mason jouant, dans Une étoile est né, un alcoolique notoire qui se suicide dans la mer. Plus tard, il interrogera même, photogramme à l'appui, la mémoire de ses films (le rôle de l'eau ?) et nous soumet deux listes de cinéastes et critiques: les morts noyés et les férus des eaux. "L'enfer c'est la piscine! » Pour son plus grand malheur critique, Luc Moullet refuse de prendre au sérieux ses références et analogies ludiques. Les complexifications de la subjectivité au cinéma ont pris une telle ampleur dans la 
théorie du cinéma, que la mise en scène frontale de Luc Moullet, lequel répond, lui aussi sans relâche, à la question, « qu'en est-il de moi maintenant dans le cinéma ? " n'est plus comprise. Les évidences de la didactique du montage et de l'effet documentaire redeviennent le propre du "sujet cinéma» dans la fiction, au-delà d'une simple thématique du film dans le film. En témoignent la double fin et la mise en scène d'un double discours contradictoire dans Anatomie d'un rapport, la mise en scène de la dialectique exploitation/exploiteur dans le documentaire social (Genèse d'un repas), le fantasme de la mort du parangon spirituel de la modernité française au cinéma ${ }^{31}$ et la mise en scène du film imaginaire dans Le Prestige de la mort.

L'atelier de cinéma de Luc Moullet est un avatar burlesque au regard de la synchronie de l'image, de la parole et du son. Luc Moullet évite la synchronie idéelle du cinéma dominant, comme son envers, la désynchronie du cinéma moderne. Il filme en faveur d'un léger décalage pour souligner l'association, le lien logique, constamment redéfini, sur le modèle pédagogique de la décomposition avant l'enchainement, commune aux danseurs, aux nageurs ou aux pianistes. Cette synchronie décalée est insolite, quasi réactionnaire quand on connaît l'engouement et l'impact des expérimentations et des essais cinématographiques qui travaillent la bande son dans le cinéma parlant et sonore depuis la période moderne (Godard, Duras, Akerman...). L'écho son/image que l'on retrouve entre le gag et la bande sonde l'inspiration cartoonesque américaine de Frank Taschlin ou de Preston Sturges ${ }^{32}$ se rejoue dans l'œuvre de Luc Moullet. Chez ce cinéaste, la voix off précède rarement la compréhension du spectateur et la voix in explique la logique d'un plan avant ou après la résolution technique (montage) ou narrative (l'histoire) du lien de son écriture. Luc Moullet est un véritable bonimenteur à l'écran de ses propres films. Cela exige de prendre au mot et à l'image ce qui semblerait n'être qu'un de ses innombrables traits d'humour. Dans Les minutes d'un faiseur de film, Luc Moullet affirme successivement «le cinéma c'est le changement ", «le cinéma c'est le striptease ", « le cinéma c'est douloureux » et « C'est chiant les extérieurs, les trucs à montrer sont toujours trop loin les uns des autres, faut bouger la caméra ». Il pointe ainsi, avec malice, tout en offrant ce qu'il faut bien appeler des illustrations à l'écran, quatre directions pour sa pédagogie du cinéma: le travestissement de l'acteur, l'incarnation individuelle, l'ardu apprentissage technique et le sens du découpage.

Anatomie d'un rapport inaugure donc une métamorphose inédite dans le mouvement de l'inscription des outils et du travail du cinéaste dans la construction et la vision du cinéma, en offrant son corps, son jeu burlesque et son visage impassible en guise de pratique de l'atelier du cinéaste à l'écran. Réfractaire à la construction de l'imaginaire de l'atelier intérieur ${ }^{33}$ romantique, l'atelier Luc Moullet ne résout pas l'aporie de la nécessité ou de l'inutilité d'un lieu de création spécifique et change avec les contingences des fictions. Cet atelier dessine les contours du projet cinématographique à partir des facultés d'adaptation au monde alentour (le cinéaste et la didactique du discours) et à travers une exposition quotidienne et pédagogique (la double fin d'Anatomie d'un rapport, le film fragmentaire du Prestige de la mort). Ni le genre intime (journal intime), ni le genre militant (documentaire politique) ne rendent compte de l'atelier de l'œuvre de Luc Moullet.

28 C'est un mouvement indécis, en équilibre entre la constitution de l'individu et son inscription sociale à partir de la médiation cinématographique offerte avec optimisme comme une médiation langagière, une pédagogie accessible à tous. Si l'autoproduction et le système d'avance sur recette sont des références explicites, Luc Moullet insiste 
également sur la confrontation des discours, l'enquête documentaire (qui commence dans différents films avec le dictionnaire, et la preuve par l'image photographique) et la joute imaginaire de la parole dans le film, comme le personnage du gendarme discute la logique et la trame narrative à l'œuvre dans Le Prestige de la mort. La théorie a raison de souligner que la porosité documentaire/fiction est inhérente au cinéma, et chez Moullet les indices pullulent : acteur-cinéaste, voix off, autofiction, réminiscence autobiographique, images du quotidien. En vrai cinéaste, Luc Moullet ne règle pas cette question une fois pour toute, définitivement. Les extraits de l'adaptation de Remèdes désespérés de Thomas Hardy (pour lequel Luc Moullet a obtenu en 2005 une subvention) dans Prestige de la mort peuvent même faire office de film de fiction dans le faux vrai documentaire.

L'apport évident de Luc Moullet dans la question de la subjectivité au cinéma adossée aux figures de l'atelier cinématographique, c'est de reprendre la croyance en l'image à zéro, sans lui faire le procès de la tromperie. La particularité de la figure de l'atelier dans le cinéma de Luc Moullet n'est ni de l'ordre du lieu de méditation et d'isolement, ni de l'attention portée aux capacités techniques, mais se constitue comme une sorte de parcours pédagogique qui se refuse à un imaginaire de l'autorité de l'art. Son univers cinématographique se situe dans une sorte de motif du bricolage (distant de la technique), à l'antipode des encombrantes figures esthétiques contradictoires de la mise en abyme de la modernité du moyen d'expression cinématographique, et du miroir du lieu d'épanouissement de l'artiste. A travers ce que la critique résiste à reconnaitre comme des fiction-documentaires, tout se passe comme si le dedans de l'artiste et le dehors de la technique comptait moins que l'instrumentalisation et l'appropriation d'un langage et, par conséquence, la naissance d'une autonomie (plutôt que d'une subjectivité) chère à une décennie (les années 70 fondatrices), en quête de cinéastes derrière leurs appareils et leurs images.

30 L'introduction de Ma première brasse est un modèle du genre pour comprendre l'atelier Luc Moullet en toute simplicité. Il s'agit d'un dispositif frontal « primitif ». Face caméra, assis sur une chaise devant un bureau en bois, dos à une fenêtre Luc Moullet nous parle :

«Je m'appelle Luc Moullet. Je suis cinéaste. J'ai 43 ans. C'est l'âge où chez les romains, on pouvait devenir sénateur. Mais on est plus chez les romains et de toute façon, je n'ai aucune espèce d'envie de devenir sénateur. A la place, pour fêter mes 43 ans, j'avais pensé à faire mon premier mouvement d'appareil de caméra. Mais je sens que c'est trop tôt, je suis pas encore capable; je préfère attendre d'avoir cinquante ans ou de faire mon dixième film. Et au lieu de faire un travelling, je voudrai apprendre à nager. Pour nous autres intellectuels, faire un nouveau film, écrire un nouveau bouquin c'est devenu de la routine. Tandis qu'avoir une nouvelle activité physique c'est quelque chose d'original, de passionnant, ça comble un manque dans notre vie. Et le jour où je saurai nager, ça sera vraiment un grand jour pour moi. Et puis, plutôt que de dépenser du fric à prendre des leçons de natation, je préfère gagner quatre briques à faire un film là-dessus. »

31 Tout est dit, si l'on considère que ce préambule humoristique est accompagné d'un jeu d'acteur grotesquement victime, Luc Moullet baissant les yeux quand il avoue ne pas être prêt. Le réalisateur prend littéralement ses distances avec la valeur accordée aux mouvements de caméra au cinéma. Le zoom avant et le recadrage sont autant de choix distinctifs de mise en scène en faveur de mouvements plus modestes.

Luc Moullet n'évoque pratiquement pas la question de la projection (en plein air, à la télévision, en DVD, ou sur n'importe quel support...) et de la diffusion ${ }^{34}$ du cinéma. Où il est question de pédagogie, il est souvent question d'optimisme et de foi, peut-être aveugle 
et hasardeuse. Pour l'anecdote, dans Anatomie d'un rapport, le personnage de Luc Moullet apprend au spectateur qu'il a profité d'une miraculeuse erreur bancaire pour faire son film. Luc Moullet a foi en l'image, une image qui ne ment pas ou qui n'aurait pas pour mission de réparer le défaut d'une autre, selon l'âpre pédagogie godardienne. Le savoirfaire de Luc Moullet revendique la sincérité de la manipulation simple des images. Acculé au constat de la difficulté matérielle à faire des films bons marchés depuis toujours, Luc Moullet refuse pourtant le drame (du fait de l'humour) ou le pathos de la vision manichéenne et immodeste (version Roger Moore) du seul contre tous. Le montage de ses plans et de ses films reprend inexorablement les $B-\grave{a}-B A$ et les premières leçons du langage cinématographique : il veut nous persuader qu'il suffit de se jeter à l'eau en évitant le plus longtemps possible son premier mouvement d'appareil de caméra (Ma première brasse) et d'entrer dans sa séquence avec « le dialogue, la fin du plan et le découpage » (Les minutes d'un faiseur de film).

La pédagogie, qui donne, d'une part un sens pratique et nous révèle un accès plus aisé que l'on ne croit, et d'autre part un guide pour les pulsions artistiques et communicationnelles du spectateur, est fait œuvre chez Luc Moullet depuis Anatomie d'un rapport. Le principe de son style minimaliste, le dénouement, est devenu une grammaire simple du cinéma jusqu'à constituer la structure principale de l'œuvre. L'atelier Moullet se construit, certes comme une résistance aux contraintes de la production, mais il mesure aussi le danger qui veut que la prégnance économique carnivore ne doive pas métaphoriquement avoir raison de l'imaginaire et de la possibilité même de réaliser. Les récentes séquences de Remèdes désespérés dans Le Prestige de la mort prennent alors valeur de symptôme de l'attention aux moyens de réaliser un film autant qu'à la liberté de le réaliser. Plutôt qu'un lieu à soi, Luc Moullet épouse la voie de la transmission didactique de ses étapes de création; plus qu'une œuvre, il propose une pédagogie cinématographique, un savoir-faire : les outils et l'échelle de l'atelier plutôt que l'atelier achevé pour lui-même.

BIBLIOGRAPHIE

\section{Filmographie :}

\section{Longs métrages :}

Anatomie d'un rapport, 1975, 16 mm, noir et blanc, 82'.

Genèse d'un repas, 1978, 16 mm, noir et blanc, 117'.

Le Prestige de la Mort, 2007, 35 mm, couleur, 75'.

\section{Court métrage :}

Ma première brasse, 1981, 16 mm, noir et blanc, 43'. 


\section{Format court pour la télévision :}

Les minutes d'un faiseur de film, Cinéma cinémas, 1983, 16 mm, noir et blanc, 13'.

Autoportrait Luc Moullet, Court Circuit, Numéro 10, (Arte), émission diffusée pour la première fois le 07.04. 2001, 04', vidéo, couleur.

\section{Bibliographie :}

Cahiers du Cinéma, « Table ronde avec la SRF [Maurice Dugowson, Pierre Kast, Jacques DoniolValcroze, Luc Moullet et Cahiers] », n³25, Spécial « Situation du cinéma français II. La Production. La technique », juin 1981, pp100-103

CERISUELO Marc, Preston Sturges ou le génie de l'Amérique, Puf, coll. Perspectives critiques, Paris, 2002.

COMBE Jean-Paul et GUITTON Hervé, " Luc Moullet en toute intimité », Rétrospective Luc Moullet à la Cinémathèque française, pp12-16.

DANEY Serge, « Un tombeau pour l'œil. (Pédagogie straubienne) » et « Le Therrorisé (pédagogie godardienne) » La Rampe. Cahier critique 1970-1982, « Le corps du cinéaste en plus (morale et engagement) 1975/78 », Gallimard, 1983, pp70-95.

DANEY Serge, Ciné-journal, Vol 1, 1981-1982, Editions des Cahiers du Cinéma, 1998, p15.

DOUCHET Jean, « Juste une jauge, donc une jauge juste », Rubrique Bonus/ Critiques, Le lecteur de DVD, Cahiers du Cinéma, novembre 2006, p97.

GARDIES André, « Le spectateur en quête du je (et réciproquement) », Le je à l'écran. Colloque de Cerisy du 14 au 21 août 1999, L'Harmattan, 2006, pp9-23.

GERSTENKORN Jacques, « De l'émergence du « je » au cinéma », La Voix du regard, $\mathrm{n}^{\circ} 12$, printemps 1999, pp6-9.

QUESTEBERT Marie-Christine, " Jeu de scène ", Des nouvelles du cinéma. Une première fois. Tome 2, ss dir Sarah Bertrand, Seuil/ Le thé des écrivains, 2004, pp144-155

LESCOURRET Marie-Anne, «L'atelier intérieur : mélancolie et création », Colloque de l'Université de Strasbourg, 4-5 mai 2006 Les espaces de l'atelier. Disponible sur Internet : http://publi-misha.ustrasbg.fr/document.php?id=119.

MOULLET Luc, « Les dispositifs du cinéma contemporain », Esprit, août/septembre 2007, pp121-129.

MOULLET Luc, « Mon travail », Trafic, $\mathrm{n}^{\circ}$ 39, automne 2001, pp16-27.

MOULLET Luc, « Les lois secrètes du Cinémargent », Le cinéma et l'argent, CRETON Laurent (ss dir), Nathan, Paris, 1999, pp45-55

MOULLET Luc, « Luc Moullet », Cinématographe, n 85, janvier 1983, pp17-19

TINEL Muriel, L'autoportrait cinématographique, Thèse, Sciences du langage, ss dir Aumont Jacques (Jury : Dominique Château, Michel de Fornel, Claude Murcia), 2004. 


\section{NOTES}

1. GERSTENKORN Jacques, «De l'émergence du «je » au cinéma », La Voix du regard, $\mathrm{n}^{\circ} 12$, printemps 1999, pp. 6-9

2. Pour mémoire, entre autres : Rebecca (1940) d'Alfred Hitchcock, Laura(1944) de Tay Garnett, Lettre d'une inconnue (1948) de Max Ophüls, Boulevard du Crépuscule (1950) de Billy Wilder, etc.

3. Selon le titre de la thèse de Muriel: TINEL Muriel, L'autoportrait cinématographique, Thèse, Sciences du langage, ss dir Aumont Jacques (Jury : Dominique Château, Michel de Fornel, Claude Murcia), 2004.

4. Au sens de Carl Gustav Jung dans Dialectique du moi et de l'inconscient (édition originale, Zurich : 1933 ; Paris : Gallimard, coll. Folio, 1986) où la persona, sur le modèle du masque de comédien, n'est pas un individu réel mais représente l'ensemble des attitudes conscientes envers le monde extérieur, soit une illusion d'individualité.

5. L'Evangile apocryphe dit de Saint Thomas, manuscrit copte daté du IV siècle découvert en 1945, près de Nag Hammadi, en Égypte, est un texte sans structure narrative. C'est un recueil de

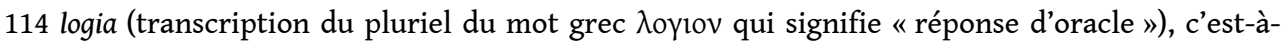
dire de paroles rapportées de Jésus. Le texte commence par : Jésus, le Vivant, les a révélées - Didyme Jude Thomas les a transcrites. "Il n'évoque ni la mort ni la résurrection de Jésus. (Source: l'introduction à L'Évangile de saint Thomas d'Helmut Kœster, Harvard, dans l'édition anglaise de l'ensemble des textes de Nag Hammadi, The Nag Hammadi Library, James Robinson, General FEd., 1977 et L'Evangile de saint Thomas de Jean Doresse, Rocher, 1988).

6. Luc Moullet anticipe d'ailleurs ce néologisme au pied de la lettre puisque dans deux de ses films où il se met en scène les bobines ont tendance à lui échapper : roulant vers une bouche d'égoût (Anatomie d'un rapport) ou quasi éparpillées sur le trottoir (Les minutes d'un faiseur de film).

7. La méfiance à l'encontre de ceux qui hypostasient leur art cinématographique comme art de l'image (la photogénie et la règle du mouvement au cinématographe de Jean Epstein par exemple) est une controverse dont Serge Daney nous donne une compréhension partiale. En effet, le critique n'a cessé de prendre partie alors qu'il lui semblait qu'une manière néfaste d'envisager le cinéma pouvait s'imposer : «L'encyclopédie du monde et la pédagogie de la perception se sont effondrés, au profit d'une formation professionnelle de l'œil, un monde de contrôleurs et de contrôlés qui communient dans l'admiration de la technique, rien que la technique.» [ Serge Daney, Cinéjournal, Vol 1, 1981-1982, Editions des Cahiers du Cinéma, 1998, p15.]

8. Non pas au sens de parti pris esthétique clos, contre lequel s'insurge un article récent de Luc Moullet, mais simple constat de la reconduction de la mise en scène frontale du burlesque qui valorise le jeu (et les déplacements) de l'acteur, les espaces et les objets comme matières premières du film.

9. «Ma weltanschauung se moul(l)e sur une ontologisation de mon Yoknapatawpha. » (extrait du dialogue du Prestige de la mort).

10. Luc Moullet a donné à Censier (Paris III) des cours d'économie du cinéma

11. Sur le célèbre modèle des articles de Serge Daney, respectivement sur Jean-Luc Godard et Jean-Marie Straub et Danièle Huillet : « Un tombeau pour l'œil. (Pédagogie straubienne) » et « Le Therrorisé (pédagogie godardienne) » réunis dans La rampe.

12. L'Hommes des roubines, Gérard Courant, 2000, Beta SP, couleur, 55 min.

13. MOULLET Luc, Mes paysages », in Cinéma, $\mathrm{N}^{\circ} 10$, automne 2005, pp. 12-19: « mon vieux deux pièces du 41 rue Lepic et une chambre chez mes parents à Roberval, dans l'Oise (Anatomie d'un rapport), retour à la rue Lepic avec Genèse d'un repas, mon studio du 27 rue Jean-pierre Timbaud, $6 e$ étage (les Minutes d'un faiseur de films), mon deux pièces du 4 bis rue Richard-Lenoir (l'Empire de Médor, Nous sommes tous des cafards, Prestige de la mort). (...) Pourquoi tournais-je 
essentiellement chez moi ? Parce que ça m'évite de me déplacer le matin et de me lever tôt, parce que je connais bien l'endroit, parce que je sais l'exploiter au mieux avant même d'écrire le script. Quand j'achète un appartement, je me demande où je vais placer la caméra avant de savoir où je mettrai mon lit. (...) la gratuité généralisée n'est pas le seul avantage. Il y a l'absence d'éclairage au profit du "tout solaire".» (p. 13).

14. MOULLET Luc, Mes paysages ", in Cinéma, $\mathrm{N}^{\circ} 10$, automne 2005, p. 16.

15. QUESTEBERT Marie-Christine, «Jeu de scène », Des nouvelles du cinéma. Une première fois. Tome 2, ss dir Sarah Bertrand, Seuil/ Le thé des écrivains, 2004, p. 148.

16. «Contrairement à d'autres cinéastes marginaux- Morder, Lehman ou Mekas, par exemplequi ont fait des choix techniques (le super 8 ou la vidéo), vous évoluez dans le système toute en restant un artisan. » : COMBE Jean-Paul et GUITTON Hervé, « Luc Moullet en toute intimité », Luc Moullet. Le contrebandier. Rétrospective Luc Moullet à la Cinémathèque française, p. 37.

17. idem, p. 16

18. MOULLET Luc, «Le martyre de San Sebastian », Cahiers du Cinéma, n 99, septembre 1959, pp36-43.

19. DOUCHET Jean, "Juste une jauge, donc une jauge juste ", Rubrique Bonus/ Critiques, Le lecteur de DVD, Cahiers du Cinéma, novembre 2006, p. 97.

20. Brigitte et Brigitte, Moullet and Cie, tournage de décembre 1965 à janvier 1966, sortie le 17 décembre 1966 au Luxembourg 1.

21. Table ronde avec la SRF [Maurice Dugowson, Pierre Kast, Jacques Doniol-Valcroze, Luc Moullet et Cahiers], Cahiers du Cinéma, $\mathrm{n}^{\circ} 325$, Spécial «Situation du cinéma français II. La Production. La technique », juin 1981, pp. 100-103

22. MOULLET Luc, « Mes paysages », in Cinéma, $\mathrm{N}^{\circ} 10$, automne 2005, p. 19.

23. COMBE Jean-Paul et GUITTON Hervé, "Luc Moullet en toute intimité ", Luc Moullet. Le contrebandier. Rétrospective Luc Moullet à la Cinémathèque française, p. 36.

24. «(...) grâce aux Cahiers, on s'apercevait qu'il y avait aussi un nouveau monde composé de nouveaux auteursqui s'ouvrait ", idem, p. 19.

25. André Gardies propose d'appeler ainsi ce qui a trait à la subjectivité dans l'énoncé, pour mieux dissocier ces «effets » des traces de subjectivité propres à la " question de l'énonciation ». GARDIES André, «Le spectateur en quête du je (et réciproquement) », Le je à l'écran. Colloque de Cerisy du 14 au 21 août 1999, L'Harmattan, 2006, pp. 9-23.

26. Le parallèle entre Luc Moullet Nani Moretti est confondant. Fondateur en 1986 de sa propre maison de production, Sacher Films, le cinéaste italien a produit les œuvres de jeunes cinéastes, tels Le Porteur de serviette de Luchetti et La Seconde fois de Calopresti dans lesquels il tient aussi le rôle principal. Apparu sous les traits d'un alter ego, Michele Apicella, faisant profession de cinéaste seulement dans un des films du personnage (Sogni d'oro, 1981) et après un détour par le documentaire avec La cosa (1990), dans Journal intime (1994) et Aprile (1998) Nanni Moretti n'éprouve plus le besoin d'utiliser un double transparent pour se présenter aux spectateurs. [Jean A. Gili, Nanni Moretti, Rome, Gremese, 2006]

27. TINEL Muriel, L'autoportrait cinématographique, Thèse, Sciences du langage, ss dir Aumont Jacques (Jury : Dominique Château, Michel de Fornel, Claude Murcia), 2004 : Chantal Akerman par Chantal Akerman (Chantal Akerman, Vidéo, 1996), Autoportrait en 7 tableaux (Olivier Fouchard, super 8, autoproduction, 1998) JLG-JLG, autoportrait de décembre (Jean-Luc Godard, 35 mm, 1995), Un film ( autoportrait) (Marcel Hanoun, vidéo, 1985), Berlin 10/90 (Robert Kramer, Béta SP, commande de télévision, 1991) et Forget me not (Unglee, 16 mm, 1979).

28. Idem, p. 123/124.

29. Dont Luc Moullet, de manière révélatrice pour l'étude de son travail, dénonce comme un système de «choix contraignants de mise en scène » dans le plus récent de ses articles : "Les dispositifs du cinéma contemporain », Esprit, août/septembre 2007, pp. 121-129. 
30. Dans un entretien avec le cinéaste, celui -ci précise: «Mon adaptation de la nouvelle de Henry James, Le Fantôme de Longstaff [1996, 16mm, couleur, $20 \mathrm{~min}$ ] était romantique. Mon adaptation de Thomas Hardy le serait certainement... (...) Pour cette photographie que vous aimez bien, j'ai donné à mon opérateur les grandes lignes. Je lui ai montré une adaptation, pas très réussie, mais où l'image est suffisamment intéressante avec une bonne composition au niveau des couleurs et de la lumière : Far from the Madding Crowd de John Schlesinger. C'était une base avec de la métrocolor. Une couleur assez chargée, assez chaude et dense. » Entretien disponible en ligne sur le site Critikat.com: http://www.critikat.com/article1373.html;[« Le prestige du rire. Entretien avec Luc Moullet à l'occasion de la sortie du Prestige de la mort » Propos recueillis par l'auteure au domicile du cinéaste le 20 juin 2007.]

31. Dans Le Prestige de la mort, dernier film de Luc Moullet à ce jour, le stratagème mis en place par le personnage de cinéaste qu'il interprète (simuler la mort pour obtenir une rétrospective et susciter une nouvelle circulation de ses œuvres en copie film) est symptomatiquement ruiné par l'effet de présence tutélaire (l'ombre et la mort ne faisant plus qu'un) du célèbre cinéaste suisse Jean-Luc Godard.

32. Marc Cerisuelo précise et développe dans son étude monographique sur le cinéaste américain ce processus d' «accomplissement du procès de cartoonisation » du cinéma de comédie, un tel mouvement doit s'entendre en incorporant la figure de Tex Avery (...) » [Marc Cerisuelo, Preston Sturges ou le génie de l'Amérique, Puf, coll. Perspectives critiques, Paris, 2002, p. 88.]

33. LESCOURRET Marie-Anne, "L'atelier intérieur: mélancolie et création", Colloque de l'Université de Strasbourg, 4-5 mai 2006 à l'auditorium du Musée d'Art Moderne et Contemporain de Strasbourg. Les espaces de l'atelier. Disponible sur Internet: http://publi-misha.u-strasbg.fr/ document.php ?id =119.

34. Dans Il Giorno della prima di' Close-Up (Nanni Moretti, 1996, 35mm, 7'), Nanni Moretti prépare la sortie du film d'Abbas Kiarostami, Close-Up, dans son cinéma de Rome, le Nuovo Sacher. Il est au téléphone avec la distributrice pour définir la publicité du film dans la presse, puis discute dans la cabine de projection avec l'opérateur et conseille les caissières sur la meilleure façon de présenter le film aux spectateurs... [ « Le prestige du rire. Entretien avec Luc Moullet à l'occasion de la sortie du Prestige de la mort » Propos recueillis par l'auteure au domicile du cinéaste le 20 juin 2007.][« Le prestige du rire. Entretien avec Luc Moullet à l'occasion de la sortie du Prestige de la mort» Propos recueillis par l'auteure au domicile du cinéaste le 20 juin 2007.]Entretien disponible en ligne sur le site Critikat.com : http://www.critikat.com/article1373.html

\section{AUTEUR}

\section{CLAUDINE LE PALLEC MARAND}

Allocataire Monitrice, Université de Paris VIII 